\title{
(2)
}

\section{CFD SIMULATION OF MULTIPHASE FLOW IN AN AIRLIFT COLUMN PHOTOBIOREACTOR}

GARCÍA S.

PATERNINA E.

PUPO O.R.

BULA A. ${ }^{*}$

Di MARE L.

\author{
Mechanical Engineering Department \\ Universidad del Norte, Km 5, Vía Puerto Colombia \\ Barranquilla Colombia
}

Received: 19/07/2014

Accepted: 24/11/2014

*to whom all correspondence should be addressed:

Available online: $18 / 12 / 2014$ e-mail: abula@uninorte.edu.co

\section{ABSTRACT}

A Computational Fluid Dynamics (CFD) simulation using CFX, ANSYS 11.0, has been carried out using a multiphase flow model with an Eulerian-Eulerian approach for an airlift column photobioreactors (PBR). Transient simulations were performed for three inlet air flow, 2, 3 and $5 \mathrm{I} \mathrm{min}^{-1}$. The contours for gas holdup, air and water velocity showed that the presence of gas phase (air bubbles) is lower in the downcomer but larger in the riser, which leads to require a vigorous mixing in the riser that will be sufficient for a continuous flow. For air, velocity vectors show that they are smaller in the downcomer than in the riser. Nevertheless, water velocity vectors are organized, pointing down in the downcomer and up in the riser. Water shear stress rate contours analysis showed that, shear stress rate regions are considerably larger in the riser, but lower in the downcomer. Due to fewer restrictions to the liquid phase in the riser, a large amount of energy is dissipated by gas liquid interactions. In the downcomer region, gas phase is almost inexistent, and so are the bubble collisions. Finally, the kinetic energy is larger at the top region of the riser, meanwhile is lower at the downcomer. Similar results are observed for energy dissipation rate.

Keywords: CFD, multi-phase flow, micro algae growth, photobioreactor, gas - liquid flow.

\section{Introduction}

Mixing applied to photobioreactors (PBR) is one the most important and critical parameters for photosynthetic microorganisms cultivation, i.e. microalgae. A mixing system for a PBR has the purpose to permit all cells to access equally and periodically the lightened zones inside the PBR (Contreras et al., 2003). Because of this, in recent years, several investigations have been developed to find an accurate hydrodynamic model to predict the complex flow regime inside an airlift PBR, taking advantage of CFD developments Optimal hydrodynamic characteristics in the process can be achieved by an efficient mixing that ensures: light absorption, homogenization, nutrients distribution, $\mathrm{CO}_{2}$ removal, gas exchange. The system also prevents cell sedimentation and thermal stratification while reducing the degree of mutual shading and the probability of photoinhibition at the illumination surfaces. (Ugwu et al., 2008; Ogbonna and Tanaka, 2000). Therefore, it is important to understand the complexity and influence that hydrodynamic behavior has over a PBR operation, since it considers transport processes such as interphase oxygen transfer,

García S., Paternina E., Pupo O.R., Bula A. and Di Mare L. (2014), CFD simulation of multiphase flow in an airlift column photobioreactor, Global NEST Journal, 16(6), 1121-1134. 
nutrient mixing and the $\mathrm{pH}$ effects. The fluid dynamics of the medium also produces the second mayor energy input to the photo bioprocess, by transferring mechanical energy to the fluid phase by some fluid circulation method (Rosello et al., 2007).

Due to the importance of mixing, pneumatically agitated reactors like airlift PBR, have turned into a very promising design alternative for microalgae cultivation, inducing a lower shear stress that does not cause mechanical damage to the cells, by using the hydro-pneumatic method (Richmond et al., 1993; Sánchez et al., 2000). The airlift PBR, which is considered the most popular modification of the bubble column reactors (Roy et al., 2006), is characterized by a bubble induced flow, with an up flow channel (riser) and a down flow channel (downcomer) (Luo and Al-Dahhan, 2008). The advantages of this type of PBR are: no moving parts, simplicity of construction, low power consumption, good mass and heat transfer characteristics, good solid suspensions, homogeneous shear stress distribution, rapid mixing, and, one of the most important, control over liquid circulation (Roy et al., 2006; Luo and Al-Dahhan, 2008; Chisti, 1998). Aeration rates and bubble diameters are controlled via air spargers which provide optimal mixing and gas transfer in the reactor (Oncel and Sukan, 2008). Some investigations (Oncel and Sukan, 2008) have favored the selection of an airlift PBR respect to bubble column because, under the same physical conditions, the presence of the draft tube in the airlift results in more effective mixing and the bubbles rising inside the draft tube provide a less turbid zone in the annulus region, thus enabling a better exposure to the light. Also in the research work of (Pupo et al., 2012), the results showed that the effects of a better mixing and light exposition greatly influenced the performance of the biological system, culture of Nannochloropsis $s p$, for obtaining a higher cell concentration in an airlift PBR, hence yielding a better cell productivity than for the bubble column PBR. The CFD simulation results confirmed that a better mixing can be achieved in an airlift PBR by the addition of the draft tube in the airlift system. Flow inside an airlift PBR is usually modeled as a multiphase flow system which consists of single phase regions bounded by moving interfaces (Manninen et al., 1996); specifically, it is considered as a two-phase flow, with a dispersed flow regime. Transport phenomena in dispersed phase (gas) flows depend upon the collective dynamics of bubbles interacting with each other and with the surrounding continuous phase (liquid) (Joshi, 2001). For developing these models, experimental investigations and numerical simulations have been used. With regard to the last ones, the use of computational tools, i.e. CFD codes, have become extensively used to analyze global and local characteristics of multiphase dynamic flow behavior in airlift and bubble column PBR (Blažej et al., 2004; Van Baten et al., 2003; Mortuza et al., 2011; Cartland et al., 2000; Studley and Battaglia, 2011). CFD can be used to optimize the photo-bioreactors geometry in order to reduce energy demands or to identify bad mixing or high shear stress zones (Rosello et al., 2007). However, it is well known that CFD simulations must be validated by experimental results before applying with accuracy in the academy or industry, particularly for multiphase applications where physics is still not fully understood (Luo and Al-Dahhan, 2011).

The objective of this work is to characterize the hydrodynamic variables of a two-phase flow system inside an airlift PBR, by using CFD multiphase simulations, with an Eulerian-Eulerian approach, looking forward for an optimization of the fluid mixing inside the airlift PBR, for microalgae cultivation purposes.

\section{Mathematical model}

To simulate a bubble induced flow inside an airlift PBR, a multiphase flow model can be used considering species transport. For this research, the Eulerian approach was chosen, which considers both, the dispersed and the continuous phases as interpenetrating continua and describes the motion for each of the two phases in an Eulerian reference frame (Luo and Al-Dahhan, 2011). This model is advantageous when considering a bubbly flow regime, since the volume fraction of the dispersed phase is very large and more bubbles must be tracked down. It is also simpler and its computational cost is also lower, however some accuracy is lost in the final results since coalescence and single bubble breakage is not considered. Never the 
less, Euler approximation is adequate and valid for modeling bubble induced multiphase flows (Studley and Battaglia, 2011; Pfleger et al., 1999; Bertola et al., 2003; Bitog et al., 2011, Krishna et al., 2000). Basic assumptions for a two-fluid model, using an Eulerian approach are the following, (Luo and Al-Dahhan, 2011):

- Both phases are considered as interpenetrating continua.

- Both fluids are considered as incompressible with a uniform pressure field: $p=p_{l}=p_{g}$

- Any small volume of the domain, at any particular time, can be considered as containing a volume fraction of gas and liquid phase.

- The dispersed gas phase is assumed as spherical bubbles with uniform size.

- Turbulence in the dispersed gas phase is insignificant, thus it is correlated to the liquid phase turbulence, which is modeled using the modified $k-\epsilon$ turbulence model.

- The whole fluid domain is considered isothermal.

- Due to Eulerian approach for the mathematical model, coalescence and bubble breakage is not taken into account. The bubble cloud is considered as a continuum with properties analogous to the liquid phase. Single bubbles are not considered (Bitog et al., 2011).

- Mass transfer between phases is not considered.

From the previous assumptions, the governing equations based on the Navier-Stoke are the following ((Luo and Al-Dahhan, 2011; Lain, 2007):

\subsection{Continuity equation}

Mass conservation is presented in Eq. (1)

$\frac{\partial(\rho r)_{\alpha}}{\partial t}+\nabla \cdot(\rho r u)_{\alpha}=0 ; \alpha=1, g$

A volume fraction constraint for both phases is imposed by Eq. (2), and the gas holdup is defined by Eq. (3) (Chisti, 1998),

$r_{1}+r_{g}=1$

$\varepsilon_{\mathrm{g}}=\frac{\mathrm{V}_{\mathrm{g}}}{\mathrm{V}_{\mathrm{g}}+\mathrm{V}_{\mathrm{l}}}$

\subsection{Momentum equation}

Momentum conservation is presented in Eq. (4)

$\frac{\partial\left(r_{\alpha} \rho_{\alpha} u_{\alpha}\right)}{\partial t}+\nabla \cdot r_{\alpha} \rho_{\alpha} u_{\alpha} u_{\alpha}=-r_{\alpha} \nabla P+r_{\alpha} \rho_{\alpha} g+\nabla \cdot\left(r_{\alpha} \mu_{\alpha, \text { eff }}\left(\nabla u_{\alpha}+\nabla u_{\alpha}^{\top}\right)\right)+M_{l, \alpha}$

The left-hand side of the equation describes the time dependent and convective terms, respectively. The right-hand side terms describe the forces acting on a fluid element (Pfleger at al., 1999). These are the overall pressure gradient, the gravitational force, the viscous stresses and the interfacial momentum forces. In the viscous stress force, the effective viscosity $\mu_{\alpha, \text { eff }}$ term includes contributions from both, the molecular viscosity $\left(\mu_{\alpha}\right)$ and the turbulent dynamic viscosity $\left(\mu_{\alpha}^{\top}\right)$ :

$\mu_{\alpha, \text { eff }}=\mu_{\alpha}+\mu_{\alpha}^{\top}$ 
The term $\mathrm{M}_{\mathrm{l}, \alpha}$, known as the interfacial momentum transfer, is statistically averaged and represents the interactions between dispersed phase and liquid phase caused by different physical phenomena, it implies that forces have the same magnitude and opposite directions for gas and liquid phase. For bubble-driven flows, the forces to be considered are: draft, lift force, virtual mass and turbulent dispersion, excluding external fields and neglecting Basset history term (Lain, 2007).

$M_{1, I}=-M_{1, g}=M_{D, I}+M_{L, I}+M_{V M, I}+M_{T D, I}$

To complete the conservation equations, the restriction over pressure is presented, which indicates that all phases share the same pressure field:

$\mathrm{P}_{\alpha}=\mathrm{P}$

\subsection{Turbulence model}

The turbulent viscosity for both, gas and liquid phase, must be taken into account, however since the density for the dispersed phase is much smaller than the density of the continuous phase, it is well-known that turbulence inside bubbles has limited effect on the liquid flow (Luo and Al-Dahhan, 2011). To calculate the dynamic viscosity of the dispersed phase, which relates its dynamic viscosity to the continuous phase viscosity, the following expression is used (Luo and Al-Dahhan, 2011):

$\mu_{\mathrm{g}}^{\top}=\frac{\rho_{\mathrm{g}}}{\rho_{\mathrm{l}}} \frac{\mu_{1}^{\top}}{\sigma}$

Where $\sigma$ stands for surface tension between liquid and gas phase. Also in a gas-liquid flow, the continuous liquid phase dynamic viscosity has two components: the shear-induced turbulence $\left(\mu_{\alpha}^{\mathrm{s}, \mathrm{T}}\right)$ and the bubble induced turbulence $\left(\mu_{\alpha}^{\mathrm{b}, \mathrm{T}}\right)$, given that turbulence could be stemmed from either the shear stresses within the continuous phases or the rising bubbles (Luo and Al-Dahhan, 2011).

$\mu_{1}^{\top}=\mu_{1}^{s, T}+\mu_{1}^{b, T}$

To calculate the shear induced dynamic viscosity several expressions have been proposed, in this paper the standard turbulent $k-\epsilon$ model proposed by Launder and Spalding is used according to (Luo and Al-Dahhan, 2011):

$\mu_{\alpha}^{\mathrm{s}, \mathrm{T}}=\mathrm{C}_{\mu} \rho_{\alpha}\left(\frac{\mathrm{k}_{\alpha}^{2}}{\epsilon_{\alpha}}\right)$

As $C_{\mu}$ represents a constant, $k_{\alpha}$ is the turbulent kinetic energy and $\epsilon_{\alpha}$ is the turbulent dissipation rate. For the last two terms, the evolution equations are presented afterward. The bubble induced turbulent dynamic viscosity is calculated according to Sato and Sekoguchi formulation presented by (Luo and Al-Dahhan, 2011):

$\mu_{\alpha}^{b, T}=0.6 \rho_{1} r_{g} d_{b}\left|u_{g}-u_{1}\right|$

The effective viscosity has three components: the molecular, turbulence shear and bubble induced turbulence viscosity.

$\mu_{1, \text { eff }}=\mu_{1}+\mu_{1}^{s, T}+\mu_{1}^{b, T}$

The $k-\epsilon$ turbulence model has been used for bubble driven flows to describe continuous phase behavior (Luo and Al-Dahhan, 2011; Lain, 2007). For liquid and gas phase: 


$$
\begin{aligned}
& \frac{\partial\left(r_{\alpha} \rho_{\alpha} k_{\alpha}\right)}{\partial t}+\nabla \cdot\left(\rho_{\alpha} r_{\alpha} u_{\alpha} k_{\alpha}\right)-\nabla \cdot\left(r_{\alpha}\left(\mu_{\alpha}+\frac{\mu_{\alpha}^{s, T}}{\sigma_{k}}\right) \nabla k_{\alpha}\right)=-r_{\alpha}\left(P_{\alpha}-\rho_{\alpha} \epsilon_{\alpha}\right)+S_{\alpha, k} \\
& \frac{\partial\left(r_{\alpha} \rho_{\alpha} \epsilon_{\alpha}\right)}{\partial t}+\nabla \cdot r_{\alpha} \rho_{\alpha} u_{\alpha} \epsilon_{\alpha}=-\nabla \cdot\left(r_{\alpha}\left(\mu_{\alpha}+\frac{\mu_{\alpha}^{s, T}}{\sigma_{\epsilon}}\right) \cdot \nabla \epsilon_{\alpha}\right)-r_{\alpha} \frac{\epsilon_{\alpha}}{k_{\alpha}}\left(c_{\epsilon 1} P_{\alpha}-c_{\epsilon 2} \rho_{\alpha} \epsilon_{\alpha}\right)+S_{\alpha, \epsilon}
\end{aligned}
$$

The production term, $\mathrm{P}_{\alpha}$, represents the turbulence production due to local shear forces in the continuous phase:

$\mathrm{P}_{\alpha}=\mu_{\alpha}^{\mathrm{s}, \mathrm{T}} \nabla \mathrm{u} \cdot\left(\nabla \mathrm{u}+\nabla \mathrm{u}^{\mathrm{T}}\right)-\frac{\mu_{\alpha}^{\mathrm{s}, \mathrm{T}}}{\rho \mathrm{Pr}_{\mathrm{t}}} \mathrm{g} \cdot \nabla \rho$

where $\operatorname{Pr}_{t}$ is the turbulent Prandtl constant which is usually approximate to 0.85 for a gas-liquid system (Luo and Al-Dahhan, 2011). The constants used in the $k-\epsilon$ model are presented in Table 1:

Table 1. Constants used in the k- $\epsilon$ model. (Zhang et al., 2006; Bertola et al., 2003)

$\begin{array}{ccccccc}\mathrm{C}_{\mu} & \mathrm{c}_{\epsilon 1} & \mathrm{c}_{\epsilon 2} & \sigma_{\mathrm{k}} & \sigma_{\epsilon} & \mathrm{K} & \mathrm{Pr}_{\mathrm{t}} \\ 0.09 & 1.44 & 1.92 & 1.0 & \frac{\mathrm{K}}{\left(\mathrm{c}_{\epsilon 1}-\mathrm{c}_{\epsilon 2}\right) \sqrt{\mathrm{C}_{\mu}}} & 0.4187 & 0.85\end{array}$

\subsection{Turbulent dispersion model}

The terms $S_{\alpha, k}$ and $S_{\alpha, \epsilon}$ are used to model additional terms, such as the turbulent dispersion. This effect describes the diffusion of gas bubbles caused by turbulent fluctuations (Pfleger et al., 1999). The gradientdiffusion model is used for the turbulent dispersion term in the continuity equation:

$\mathrm{S}_{\alpha}=\nabla\left(\Gamma_{\alpha} \cdot \nabla r_{\alpha}\right)=\nabla\left(D_{\alpha} \cdot \nabla\left(\rho_{\alpha} r_{\alpha}\right)\right)$

The dispersion coefficient of a two-phase flow $\Gamma_{\alpha}$ is defined using turbulent viscosity and the turbulent Prandtl number (Pfleger et al., 1999):

$\Gamma_{\alpha}=\frac{\mu_{\alpha}^{s, T}}{\operatorname{Pr}_{t}}$

\section{Experimental setup}

Figure 1 shows the draft tube airlift PBR where experiments were carried out. The PBR was made of acrylic with a thickness of $4 \mathrm{~mm}$, a height of $750 \mathrm{~mm}$, an external column diameter of $250 \mathrm{~mm}$, a draft tube diameter of $150 \mathrm{~mm}$ and 4 windows located near the top of the draft tube to allow water circulation. A CAD model was designed using Solidworks ${ }^{\circledR} 2010$ and it is presented in Figure 2. The spherical porous sparger used to supply the air is located at the bottom of the PBR and has $40 \mathrm{~mm}$ in diameter. The experiments were

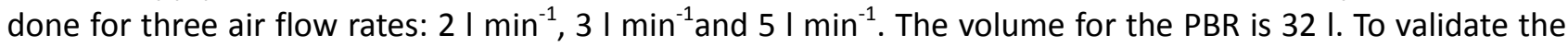
behavior of the two phase flow in the PBR, a high-resolution video-camera was used to track the flow pattern of the bubbles and the fluid. 




Figure 1. Experimental airlift photobioreactor

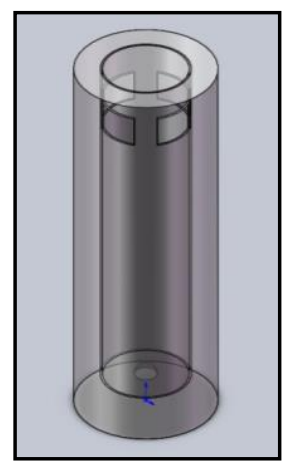

Figure 2. Photobioreactor CAD model

\section{Computational model configuration}

The governing equations presented in the previous section, represent the mathematical model that CFX Ansys ${ }^{\circledR} 11.0$ computational package uses to solve numerically the continuity balances for mass, momentum, turbulent energy and the additional closure terms. This software uses a finite volume code for discretizing Navier-Stoke equations into a group of lineal equations.

\subsection{Mesh configuration}

Once the computational domain was defined, a volumetric mesh was established using the parameters shown in Table 2. Also an adaptive mesh refinement was done to improve mesh resolution in regions where the fluid boundary layer develops. A grid independence analysis was performed and the final statistics are shown in Table 3.

Table 2. Inflation function parameters

\begin{tabular}{lc}
\hline Option: & Smooth transition \\
\hline Location: & 3 lateral faces \\
\hline Transition Rate: & 0.77 \\
\hline Maximum Layers: & 5 \\
\hline Growth Rate: & 1.3 \\
\hline
\end{tabular}

Table 3. Volumetric mesh final statistics

\begin{tabular}{|c|c|}
\hline Nodes: & 356,012 \\
\hline Elements: & $1,230,217$ \\
\hline - Tetrahedron: & 843,815 \\
\hline - $\quad$ Triangular prisms (wedges) & 383,299 \\
\hline - $\quad$ Pyramids & 3,103 \\
\hline
\end{tabular}

The final refinement for the volumetric mesh is shown in Figure 3 and 4.

Figure 5 and figure 6 show the isometric and lateral view of the PBR volume mesh. The mesh used is an unstructured grid, in which the elements and nodes do not have specific order (they cannot be directly identified by their indexes $i, j, k$ ). Unstructured grids are advantageous compared to structured grids, since tetrahedrons can be generated regardless of the complexity of the domain (Sanchez, 2004), and the computational time required for generating the grids is shorter. 


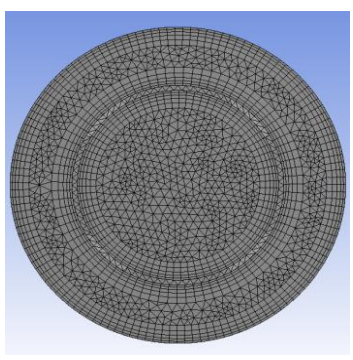

Figure 3. Top View

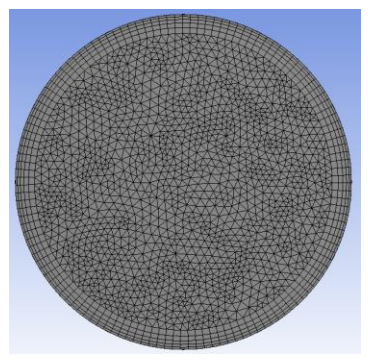

Figure 4. Bottom View

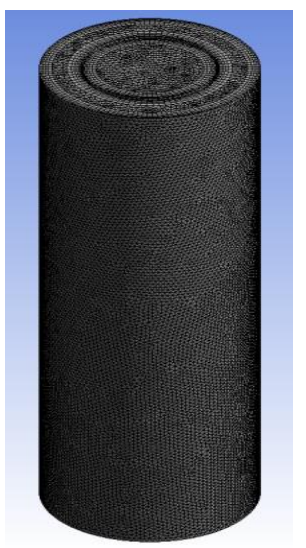

Figure 5. Isometric View

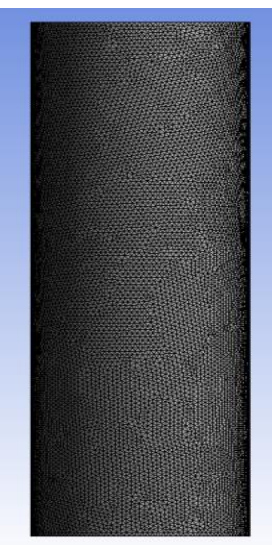

Figure 6. Lateral view

\subsection{Particle model configuration}

For the configuration of the particle model, which indicates that interfacial momentum transfer is directly dependent on superficial contact area between the two phases; water is chosen as the continuous fluid and air as the dispersed fluid, with a mean bubble diameter of $4 \mathrm{~mm}$ and a buoyancy reference density of 1.22 $\mathrm{kg} \mathrm{m}^{-3}$.

An isothermal model has been considered for the fluid, with $25^{\circ} \mathrm{C}$, thus no temperature gradient exists inside the domain. The $k-\epsilon$ model is chosen for turbulence modeling and for the buoyancy turbulence; the two additional terms for production and dissipation are included. Density difference is specified as the buoyancy model for each of the two fluids. Regarding the interfacial momentum transfer, the coefficients are presented in Table 4 (Zhang et al., 2006).

Table 4. Interfacial momentum transfer coefficients

\begin{tabular}{lc} 
Momentum transfer forces & Coefficients \\
\hline Drag force & $C_{D}=0.98$ \\
\hline Lift force & $C_{L}=0.5$ \\
\hline Virtual mass force & $C_{V M}=0.5$ \\
\hline Turbulent dispersion force & $C_{T D}=0.3$ \\
\hline
\end{tabular}

\subsection{Initial Conditions}

Turbulence was set to low intensity because the system has not been perturbed yet. Water velocity is established as $V_{1}=0 \mathrm{~m} / \mathrm{s}$ (fluid is stationary) and volume fraction to $r_{1}=1$ (liquid phase occupies the entire domain). Air velocity is determined by the mass flow supplied to the system from the porous sparger, this value was variable. And air volume fraction was set to zero.

\subsection{Boundary Conditions}

The inlet condition is placed at the surface of the sparger (semi-sphere shell), the flow regime is subsonic, turbulence is specified in medium intensity, air mass flow is set into a nominal value (inlet variable) and the volume fraction to one (gas occupies the entire fraction). In the other hand, water mass flow and volume fraction are both zero. The outlet boundary, which is located at the top of the PRB, is established as a degassing condition, meaning that this is a free surface where disperse bubble may leave the system, but not the water. Using this condition the air mass balance can be achieved. The remaining boundaries of the model are set as wall boundary conditions, with non-slip surface and smooth roughness wall characteristics. 


\section{Results}

The transient simulation was performed for $309.2 \mathrm{~s}$, and it was divided in 646 steps. The total simulation time was defined to allow sufficient time for the liquid flow pattern to form and the bubble plume to oscillate inside the PBR.

Five minutes were considered for the transient simulation. The initial time steps were smaller in order to obtain the largest amount of computational information at the beginning of the transient process where air is supplied and the bubble plume is formed. The numbers of steps used are the following: 4 steps of $0.05 \mathrm{~s}$, 10 steps of $0.1 \mathrm{~s}, 32$ steps of $0.25 \mathrm{~s}$, and 600 steps of $0.5 \mathrm{~s}$. The results are presented in the spatial domain of the PBR projected on XY plane. Air mass flow was changed between $0.5-5 / \mathrm{min}^{-1}$. Is it important to emphasize that flow moves mainly on axial direction ( $Y$ axis). Figure 7 gas holdup contours shows that the higher volume fraction region is close to the sparger, reaching a maximum of , $\alpha_{g}=0.055$.

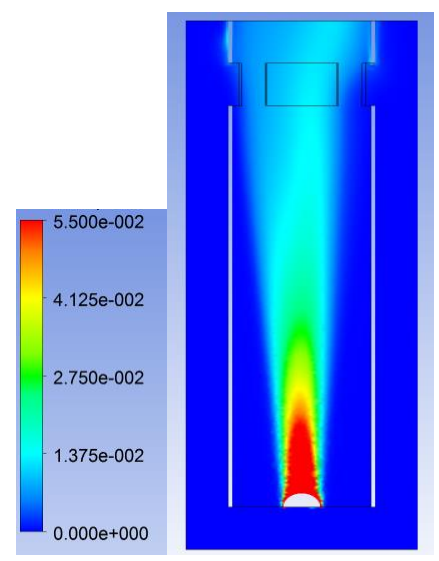

Figure 7. Gas holdup contour $\left(2 \mid \mathrm{min}^{-1}\right)$

\subsection{Water and air velocity contours}

Figure 8 shows air superficial velocity contours. An analogous behavior respect to gas holdup is noticed, where the higher velocity region is around the sparger, and the maximum magnitude is $0.04 \mathrm{~m} \mathrm{~s}^{-1}$. This result was expected due to close relation of these variables.

Figure 9 presents air velocity contours. It can be observed that the region with the highest velocity is in the middle of the riser, where bubble plume oscillates (red color zone). As was also obtained for the air superficial velocity contour, air velocity is close to zero in the downcomer, where the existence of dispersed bubbles (gas phase) is very low.

In both contours it is evident how gas phase (dispersed bubbles) occupies, in general, just the riser region and gas fraction and air superficial velocity magnitudes diminish from the bottom to the top of the column, where due to the degasification condition, bubbles virtually come out of the system to achieve the mass balance. Because of this, gas volume fraction is near zero in the downcomer, and so is the air superficial velocity, due to close absence of gas phase in this region. The behavior of the flow flied for these two variables is very similar. These results are as expected and in accordance to the work in (Luo and Al-Dahhan, 2008). It is important to underline that while running the experimental tests in the PBR, small bubbles that crashed or exploited in the upward path where observed. Other bubbles coalesced and not many reach the PBR windows to re-circulate. Then the existence of the gas phase in the downcomer region is very poor, where the fluid comes down due to the movement generated by the bubbles. 


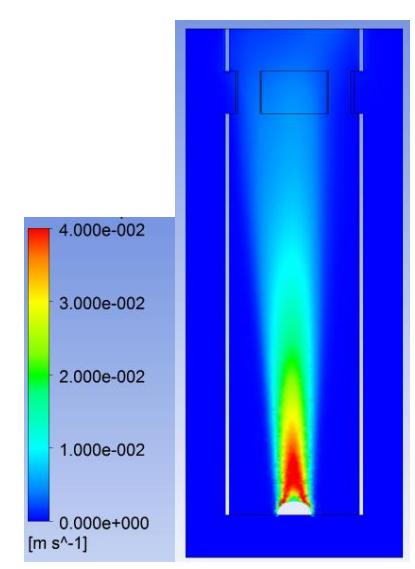

Figure 8. Air superficial velocity contour $\left(2 \mathrm{I} \mathrm{m}^{-1}\right)$

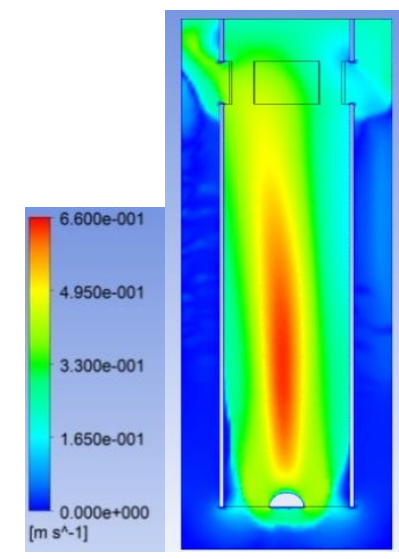

Figure 9. Air velocity contour $\left(2 \mathrm{Imin}^{-1}\right)$



Figure 10. Water velocity contour $\left(2 \mid \mathrm{min}^{-1}\right)$

Figure 10 presents water velocity contours. These velocity magnitudes are even lower than gas phase velocity, since the liquid phase is driven by the bubble flow. Also the highest water velocity is in the riser, while the lowest is in the downcomer. The velocity contours at the windows show the water leaving the raiser and coming into the downcomer. Also at the window, the liquid velocity magnitude is very low. Furthermore, there is water circulation on the top of the outer cylinder. This is one the most significant facts in relation to mixing optimization inside the PBR.

\subsection{Water and air velocity vectors}

The behavior of the air and water velocity fields inside the PBR structure IS analyzed from the velocity vectors projections on the $\mathrm{XY}$ plane.

Figure 11 shows air velocity vectors coming out of the computational domain due to the degassing condition at the outlet boundary, which forces air to flow out of the column for an appropriate mass balance. This condition allows modeling a free surface, where dispersed bubbles can escape from the domain, but not the liquid phase. By using this condition, air mass balance is completed, the same amount of air flow that enters the domain then leaves. In the downcomer of the PBR, some small air velocity vectors can be observed, which indicates that some amount of air mass is dragged by the water.

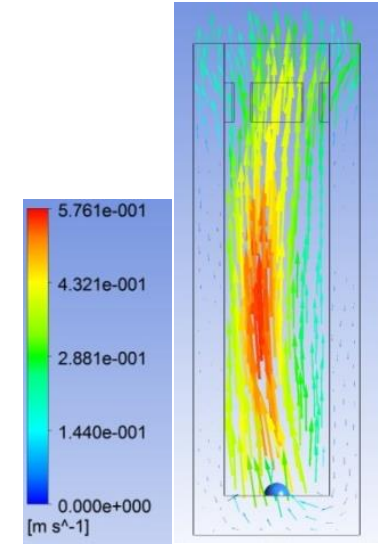

Figure 11. Air velocity vectors in the PBR $\left(2 \mid \mathrm{min}^{-1}\right)$

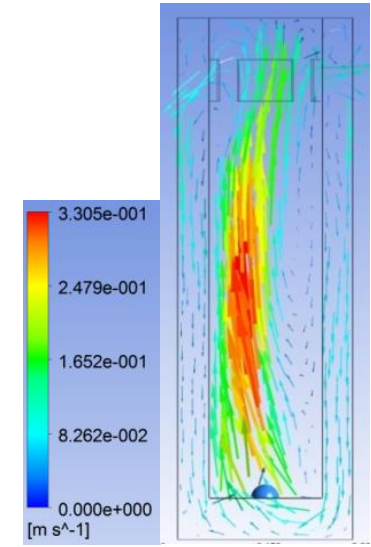

Figure 12. Water velocity vectors in the PBR $\left(2 \mid \mathrm{min}^{-1}\right)$ 
At the bottom of the column some vectors can also be noticed, this shows that some recirculation toward the riser can be achieved. In accordance to the air velocity and gas holdup contours, air velocity in the riser is always higher.

Water velocity vectors are presented in Figure 12. They represent the mixing level in the PBR domain and, since liquid phase occupies the whole PBR volume and must drive the microalgae to continuous circulation. It is also noticed that, for this specific condition of $2 \mathrm{I} \mathrm{min}^{-1}$, a regular and constant circulation of the fluid can be obtained. It is evident that velocity vectors are well organized, pointing down in the downcomer and flowing up to the riser due to eddy turbulence effects that generate low pressure zones.

It is noticed that the bubble plume is not centered but located in the left side of the column and no stagnation regions are observed, which is essential for achieving a good mixing. It is important to remark that back-circulation is not symmetrically distributed, being stronger and located in the opposite side of the plume, due to the generation of low pressure zones dragging the fluid. As discussed in (Pupo et al., 2012), a better mixing can be achieved in an airlift PBR due to the addition of the draft tube in the system.

\subsection{Shear stress rate contours}

The magnitude of the hydraulic forces in the flow is analyzed by the shear stress rate variable. These parameters are important for designing and operating bioreactors for microalgae culture. High shear stress can cause damage to the microorganism cells, and must be avoided. Never the less, there is not enough quantitative information about the shear stress field in airlift reactors, largely due to the lack of suitable measurement techniques (Luo and Al-Dahhan, 2008).

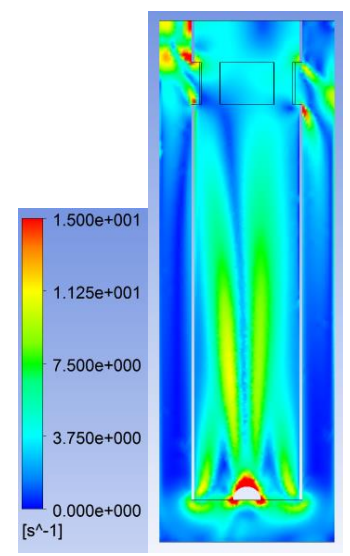

Figure 13. Water shear stress rate contour $\left(2 \mid \mathrm{min}^{-1}\right)$

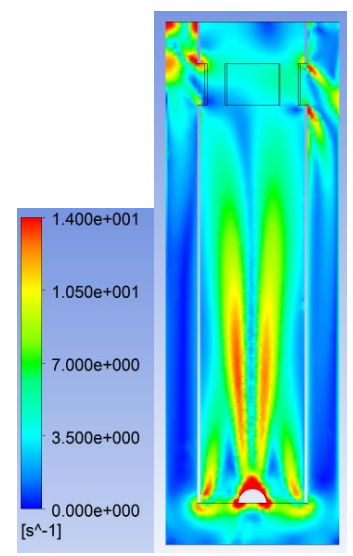

Figure 14. Water shear stress rate contour $\left(3 \mid \mathrm{min}^{-1}\right)$

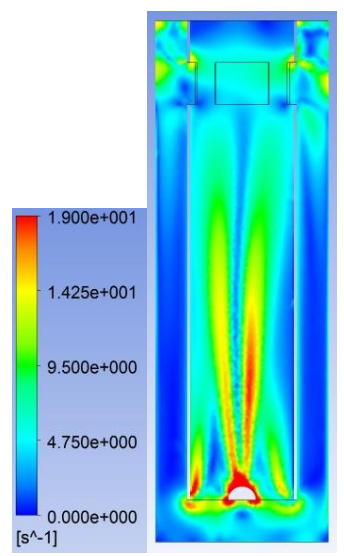

Figure 15. Water shear stress rate contour $\left(5 \mid \mathrm{min}^{-1}\right)$

Due to that, many researchers have used dimensional analysis and tried to empirically correlate shear stress with operating conditions (superficial gas velocity). However, many of these correlations are invalid since turbulence in the reactor depends on the power input or superficial gas velocity, and the momentum transport properties of the fluid (Chisti, 1998; Luo and Al-Dahhan, 2008). Figures 13, 14 and 15 show the water shear stress contours, since water is the predominant phase in the system and generates most of the stress in the potential microalgae cell. Results were obtained for the following air flows: $2,3,5 \mathrm{I} \mathrm{min}^{-1}$. The three cases show that shear stress regions are significantly higher in the riser, where water velocity is higher, while in the other regions, especially in the downcomer, the shear stress magnitude remains low, almost absent due to the a low velocity. The previous facts is consistent, because in the riser the liquid flow is least confined by the reactor column walls, and a large amount of energy is dissipated by gas-liquid collisions, while in the downcomer the gas holdup is lower. These results are in accordance with (Luo and Al-Dahhan, 2008). 


\subsection{Turbulent kinetic energy and turbulent dissipation rate contours}

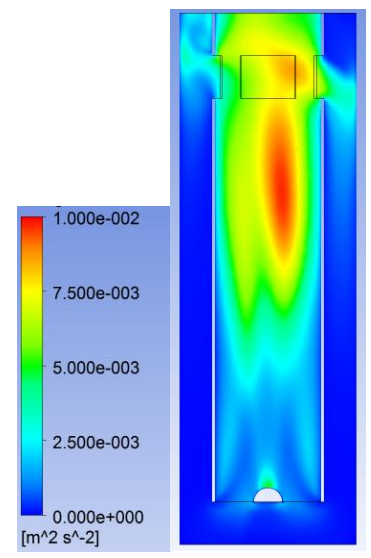

Figure 16. Water turbulent kinetic energy contour $\left(2 \mid \mathrm{min}^{-1}\right)$



Figure 17. Water turbulent eddy dissipation rate contour $\left(2 \mid \mathrm{min}^{-1}\right)$

Turbulent kinetic energy and turbulent dissipation rate for water phase are also two important variables for studying and quantifying the liquid mixing level in the multiphase flow inside the PBR. These variables are directly related to $k-\varepsilon$ turbulence model used. Similar to the shear stress rate variable, the lack of information has lead several researchers to attribute empirical values to eddy turbulent diffusitvity instead of evaluated experimental data. For this paper turbulent kinetic energy and turbulent dissipation rate contours are presented, which allow global visualization of this variables for the liquid phase. Figure 16 and Figure 17 show that the highest magnitude of the kinetic energy contour is observed in the top region of the riser, meanwhile in the downcomer the kinetic energy is low, which again confirms that gas-liquid interaction has influence on kinetic energy distribution and similarly also on the dissipation rate.

\section{Experimental validation}

To carry out the experimental validation in the PBR, a particle tracking method was adapted using a high resolution digital camera. A small amount of artemias, which are a genus of aquatic crustaceans known as brine shrimp (its size is lower than $500 \mu \mathrm{m}$ ), was supplied to the system, in order to track the artemias circulation flow and then to approximately calculate the water velocity, considering a margin of error. For this experimental setup, the external column was divided in 4 segments by marking its walls. Each section had $0.1 \mathrm{~m}$ high also the first division was $10 \mathrm{~cm}$ above the sparger, where the bubble plume is more visible. The time required for a group of these particles (artemias) to reach the marks was registered during the ascending trajectory. Afterward, the approximate velocity of these particles was calculated. This experiment was repeated several times to have a sufficient data to analyze it. Once the velocities were obtained, they were averaged for each segment to estimate the liquid phase velocity in each section. Results are shown Table 5. It is important to keep in mind that the experimental water velocity must be lower than the actual velocity, since the particle has a lower velocity than the water itself.

Table 5. Water velocity

$\begin{array}{cccc}\text { Segment } & \text { Measured Velocity }\left(\mathrm{m} \mathrm{s}^{-1}\right) & \text { CFD simulated Velocity }\left(\mathrm{m} \mathrm{s}^{-1}\right) & \text { Error, \% } \\ 4 & 0.456 & 0.42 & 8 \\ 3 & 0.527 & 0.47 & 11 \\ 2 & 0.567 & 0.49 & 14 \\ 1 & 0.651 & 0,49 & 25\end{array}$


To reassure the validity and coherence of the simulation results, several time frames of the video were compared with the computational transient results. As an illustration, Figures 18 and 19 show that the results, at 1 and 100 seconds of simulation time. Some other procedures are presented in previous investigations (Michele and Hempel, 2002)

At $t=1 \mathrm{~s}$, air bubbles were beginning to lift inside the draft tube and for $t=100$, bubble plume was oscillating. The behavior is similar for both, the experimental and the computational system, proving that the dynamic of the real fluid flow reflects on the oscillations of the computational two-phase flow. These results were shown in a previous article (Garcia et al., 2012).
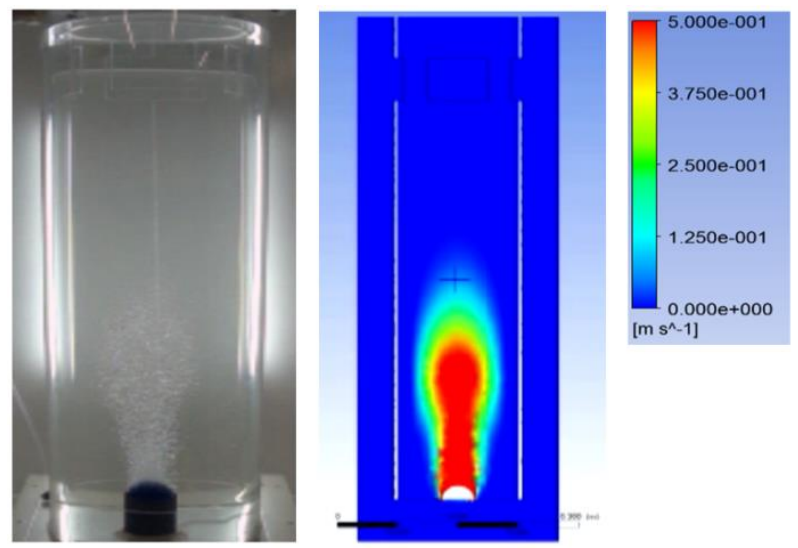

Figure 18. PBR experimental and CFD results at $\mathrm{t}=1 \mathrm{~s}$
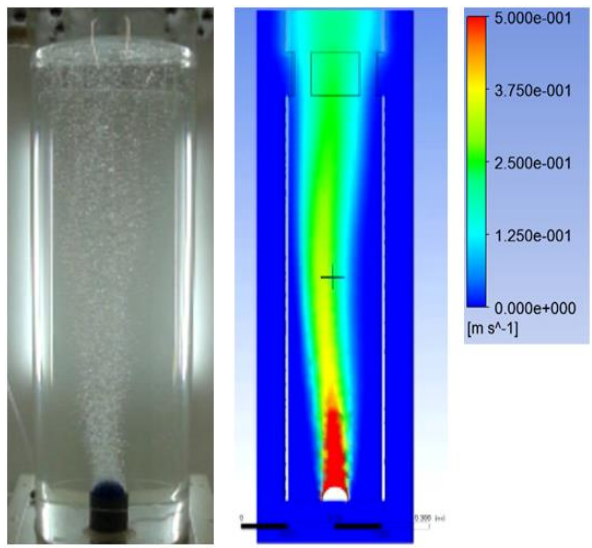

Figure 19. PBR experimental and CFD results at $\mathrm{t}=100 \mathrm{~s}$

\section{Conclusions}

Based on the simulation results for the steady and transient state of the two-phase flow, it can be concluded that Eulerian approach and the mathematical model for multiphase flow, allowed the correct characterization of the hydrodynamic behavior of the two-phase flow (water-air bubbles) inside the PBR domain.

Respect to the gas holdup, it was observed that the presence of the gas phase is lower in the downcomer but bigger in the riser. This behavior leads to the need of generating a vigorous mixing in the riser that will be enough for the liquid flow to induce the particles (e.g. microalgae cells) to a continuous recirculation in the downcomer, by the gravity force. This region is even more important in photosynthetic biomass culture applications, like it was the final purpose of the PBR construction, where flow is naturally more static, which permits the cells a longer residence time, then a longer exposition to the light.

Comparing water shear stress rates, it can be observed that independently from the air flow, the shear stress rate is significantly higher in the riser, and lower in the downcomer. This is because the liquid flow in the riser is less confined by the column walls, so a large amount of energy is dissipated by gas liquid interactions. On the other hand, in the downcomer, gas phase is almost absent, and so are the collisions of bubbles.

In particular, the selection of the $k-\epsilon$ model was adequate for a general description of the turbulence effects in the flow field. Averaged values for the turbulent kinetic energy and the dissipation rate were obtained, both variables showed that at the top of the riser the turbulence effects are greater, but lower in the downcomer, meaning that the gas-liquid interactions had influence on kinetic energy distribution and similarly also on the dissipation rate. However, it is important to explain that physical phenomena like, micro-eddies formation can not be visualized in the simulation results, although its effects permit the 
recirculation of liquid flow at the bottom of the dowcomer. Using a more advanced and complex model like the LES (Large Eddy Scale) might lead to a deeper analysis of the turbulence phenomenon of multiphase flow.

The experimental validation, despite a nonconventional technique, allowed verifying the oscillatory nature of the two-phase flow in the riser, and the poor presence of gas phase in the downcomer. Finally it can be stated that CFD is useful for characterizing and predicting the behavior of bubble induced flow inside a PBR under different mixing conditions, and optimizing the fluid mixing inside this type of photobioreactors.

\section{Nomenclature}

$\begin{array}{llll}\mathrm{d}_{\mathrm{B}} & \text { Bubble diameter } & \mathrm{r} & \text { Volume fraction } \\ \mathrm{g} & \text { Gravity } & \mathrm{S}_{\alpha, \mathrm{k}} / \mathrm{S}_{\alpha, \varepsilon} & \text { Source terms } \\ \mathrm{M}_{\mathrm{l}, \alpha} & \text { Momentum force at the interphase for } \alpha \text { phase } & \mathrm{t} & \text { Time } \\ \mathrm{P}_{\alpha} & \text { Turbulent kinetic energy production term } & \mathrm{u} & \text { Phase velocity } \\ \mathrm{Pr}_{\mathrm{t}} & \text { Turbulent Prandtl } & \mathrm{V}_{\mathrm{g}}, \mathrm{V}_{\mathrm{l}} & \text { Gas and liquid volume in the reactor }\end{array}$

\section{Greek symbols}

$\alpha \quad$ Phase (liquid/gas)

$\varepsilon \quad$ Turbulence energy dissipation rate

$\varepsilon_{\mathrm{g}} \quad$ Gas holdup

$\mathrm{k} \quad$ Turbulence kinetic energy

\section{Subsscripts}

$\begin{array}{ll}\text { I } & \text { Liquid phase } \\ g & \text { Gas phase } \\ \text { I } & \text { Intherphase force } \\ \text { D } & \text { Draft }\end{array}$

$\begin{array}{ll}\rho & \text { Density } \\ \nabla P & \text { Pressure gradient } \\ \sigma & \text { Surface tension } \\ \Gamma_{\alpha} & \text { Dispersion Coefficient }\end{array}$

$\begin{array}{ll}\text { L } & \text { Lift } \\ \text { VM } & \text { Virtual mass } \\ \text { TD } & \text { Turbulent dispersion }\end{array}$

\section{Acknowledgements}

The authors want to acknowledge COLCIENCIAS-UNINORTE for the support obtained from the YOUNG RESEARCHER project: "Diseño de fotobiorreactor para optimizar la producción de microalgas" and to the Aquaculture Research Institute CENIACUA for contributing with their facilities and personnel to carry out the microalgae cultivation.

\section{References}

Bertola F., Vanni M. and Baldi G. (2003), Application of Computational Fluid Dynamics to Multiphase Flow in Bubble Columns, Int J Chem React Eng., 1, A3, 1-16.

Bitog J., Lee I., Lee C., Kim K., Hwang H., Hong S., Seo I., Kwon K. and Mostafa E. (2011), Application of computational fluid dynamics for modeling and designing photobioreactors for microalgae production: A review, Comput Electron Agr., 76, 131-147.

Blažej M., Cartland Glover G., Generalis S. and Markoš J. (2004), Gas-liquid simulation of an airlift bubble column reactor, Chem Eng Process., 43, 137-144.

Cartland Glover G., Generalis S. and Thomas N. (2000), CFD and Bubble Column Reactors: Simulation and Experiment, Chem Pap., 54(6a), 361-369.

Chisti Y. (1998), Pneumatically Agitated Bioreactors in Industrial and Environmental Bioprocessing: Hydrodynamics, Hydraulics, and Transport Phenomena, Appl Mech Revs., 51(1), 33-112.

Contreras-Flores C., Peña-Castro J., Flores-Cotera L. and Cañizares-Villanueva R. (2003), Avance en el diseño de fotobiorreactores para el cultivo de microalgas, Interciencia, 28(8), 450-456.

García S., Pupo O., Paternina E., Bula A. and Acuña F. (2012), CFD Simulation of multiphase flow in an airlift column photobioreactor for the cultivation of microalgae. ASME 2012 6th International Conference on Energy Sustainability \& 10th Fuel Cell Science, Engineering and Technology Conference, 1-10.

Joshi J. (2001), Computational flow modeling and design of bubble column reactors, Chem Eng Sci., 56, 5893-5933. 
Krishna R., van Baten J. and Urseanu M. (2000), Three-phase Eulerian simulations of bubble column reactors operating in the churn-turbulent regime: a scale up strategy, Chem Eng Sci., 55(16), 3275-3286.

Lain S. (2007), Modelado y simulación de flujos inducidos por burbujas, Universidad Autónoma de Occidente, Cali, Colombia. 1-183.

Luo H. and Al-Dahhan M. (2008), Local characteristics of hydrodynamics in draft tube airlift bioreactor, Chem Eng Sci., 63(11), 3057-3068.

Luo H. and Al-Dahhan M. (2008), Macro-mixing in a draft-tube airlift bioreactor, Chem Eng Sci., 63, $1572-1585$.

Luo H. and Al-Dahhan M. (2011), Verification and validation of CFD simulations for local flow dynamics in a draft tube airlift bioreactor, Chem Eng Sci., 66, 907-923.

Manninen M., Taivassalo V. and Kallio S. (1996), On the mixture model for multiphase flow. Espoo, Finland: VTT Publications, 288, 1-67.

Michele V. and Hempel D. (2002), Liquid flow and phase holdup - measurement and CFD modeling for two-and threephase bubble columns, Chem Eng Sci., 57, 1899-1908.

Mortuza S., Kommareddy A., Gent S. and Anderson G. (2011), Computational and experimental investigation of bubble circulation patterns within a column photobioreactor. Proceedings of the 5th International Conference on Energy Sustainability \& 9th Fuel Cell Science, Engineering and Technology Conference. 1-10.

Ogbonna J. and Tanaka H. (2000), Light requirement and photosynthetic cell cultivation - Development of processes for efficient light utilization in photobioreactors, J Appl Phycol., 12, 207-218.

Oncel S. and Sukan F. (2008), Comparison of two different pneumatically mixed column photobioreactors for the cultivation of Artrospira platensis (Spirulina platensis), Bioresource Technol., 99, 4755-4760.

Pfleger D., Gomes S., Gilbert N. and Wagner H. (1999), Hydrodynamic simulations of laboratory scale bubble columns fundamental studies of the Eulerian-Eulerian modeling approach, Chem Eng Sci., 54, 5091-5099.

Pupo O., García S., Paternina E., Bula A., Cortina A. and Acuña F. (2012), Comparison of Two Different Vertical Column Photobioreactors for the Cultivation of Nannochloropsis sp., J Energ Resour, 135(1), 7 pages.

Richmond A., Boussiba S., Vonshak A. and Kopel R. (1993), A new tubular reactor for mass production of microalgae outdoors, J Appl Phycol., 5, 327-332.

Rosello Sastre R., Csögör Z., Perner-Nochta I., Fleck-Schneider P. and Posten C. (2007), Scale-down of microalgae cultivations in tubular photobioreactors - A conceptual approach, J Biotechnol., 132, 127-33.

Roy S., Dhotre M. and Joshi J. (2006), CFD Simulation of flow and axial dispersion in external loop airlift reactor, Chem Eng Res Des., 84, A8, 677-690.

Sanchez J. (2004), Parallel Direct Numerical Simulation of Navier-Stokes boundary layer equations for detached flows. Applications in tiered decks sport facilities, School of Engineers of Seville, Seville, Spain, (In Spanish).

Sánchez Mirón A., García Camacho F., Contreras Gómez A., Molina Grima E. and Chisti Y. (2000), Bubble-Column and Airlift Photobioreactors for Algal Culture, AIChE J., 46, 9, 1872-1887.

Studley A.C and Battaglia F. (2011), CFD analyses of the mixing characteristics in bubble column and airlift reactors. Proceedings of the ASME 2011 Int. Mechanical Engineering Congr. \& Expo., Denver, Co. 1-10.

Van Baten J., Ellenberger J. and Krishna R. (2003), Hydrodynamics of internal air-lift reactors: experiments versus CFD simulations, Chem Eng Process., 42, 733-742.

Ugwu C., Aoyagi H. and Uchiyama H. (2008), Photobioreactors for mass cultivation of algae, Bioresource Technol., 99, 4021-4028.

Zhang, D., Deen N. and Kuipers J. (2006), Numerical simulation of the dynamic flow behavior in a bubble column: A study of closures for turbulence and interface forces, Chem Eng Sci., 61, 23, 7593-7608. 